\title{
The analytic solution of near-tip stress fields for perfectly plastic pressure-sensitive material under plane stress condition
}

\author{
F.Z. LI \\ Department of Mechanical Engineering and Applied Mechanics, The University of Michigan, Ann Arbor, Michigan 48109, \\ USA; Current address: AC Rochester Division, General Motors Corporation, 1300 N. Dort Highway, Flint, MI 48556, \\ USA.
}

Received 5 February 1990; accepted in revised form 15 March 1991

\begin{abstract}
Alstract. Different from dense metals, many engineering materials exhibit pressure-sensitive yielding and plastic volumetric deformation. Adopting a yield criterion that contains a linear combination of the Mises stress and the hydrostatic stress, the analytic solutions of plane-stress mode I perfectly-plastic near-tip stress fields for pressuresensitive materials are derived. Also, the relevant characteristic fields are presented. This perfectly plastic solution, containing a pressure sensitivity parameter $\mu$, is shown to correspond to the limit of low-hardening solutions, and when $\mu=0$ it reduces to the perfectly plastic solution of near-tip fields for the Mises material given by Hutchinson [1]. The effects of material pressure sensitivity on the near-tip fields are discussed.
\end{abstract}

\section{Introduction}

Classical plasticity theory based on the Von Mises yield criterion assumes that the hydrostatic pressure has no effect on material plastic deformation, and hence plastic dilatancy is neglected. This theory is applicable mainly to dense metals. Different from dense metals, many engineering materials exhibit pressure-sensitive yielding and plastic volumetric deformation. They include rocks, concretes, soils, other porous materials and two important advanced materials toughened polymer and ceramics. Experimental results and detailed discussion concerning the mechanical behavior of these materials can be found in Drucker [2], Spitzig and Richmond [3], Carapellucci and Yee [4], Sue and Yee [5], and Chen and Reyes Morel [6].

We adopt a simple pressure-sensitive yielding criterion that contains two stress invariants, the effective shear stress $\bar{\tau}_{e}$ and the hydrostatic stress $\bar{\sigma}_{m}$. The yield criterion is stated as

$$
\psi\left(\bar{\sigma}_{i j}\right)=\bar{\tau}_{e}+\mu \bar{\sigma}_{m}=Q
$$

where $\bar{\tau}_{e}=\left(\bar{s}_{i j} \bar{s}_{i j} / 2\right)^{1 / 2}, \bar{s}_{i j}=\bar{\sigma}_{i j}-\bar{\sigma}_{m} \delta_{i j}, \bar{\sigma}_{m}=\bar{\sigma}_{k k} / 3$, and $\psi\left(\bar{\sigma}_{i j}\right)$ represents the current yield surface in the stress space. The material constant $\mu$ measures the pressure sensitivity at yield. The characteristic yield strength $Q$ can be taken to depend on the plastic work $W^{p}$.

A direct measurement of the pressure sensitivity factor $\mu$ relies on shear experiments under pressure. It can be obtained from the difference between the compressive yield strength $\bar{\sigma}_{c}$ and the tensile yield strength $\bar{\sigma}_{t}$ through the relation (Needleman and Rice [7])

$$
\mu=\sqrt{3} \frac{\bar{\sigma}_{c}-\bar{\sigma}_{t}}{\bar{\sigma}_{c}+\bar{\sigma}_{t}}
$$

An alternative method to determine $\mu$ is to perform compressive tests under pressure $\bar{p}$. Let $\bar{\sigma}_{c}^{0}$ denote the compressive yield strength in the absence of pressure, and $\bar{\sigma}_{c}^{p}$ denote the compressive 
yield strength when superimposed by hydrostatic pressure $\bar{p}$. If the experimental data can be fitted by the linear relation [6] $\bar{\sigma}_{c}^{p}=\bar{\sigma}_{c}^{0}+\psi \bar{p}$, the factor $\mu$ can be calculated according to

$$
\mu=\sqrt{3} \frac{\psi}{3+\psi}
$$

Note that the relations (1.2) and (1.3) give the same upper bound of $\mu$, equal to $\sqrt{3}$. The experimental curves in [4] show that the factor $\mu$ for glassy bisphenol A-polycarbonate is about 0.14 . For $\mathrm{ZrO}_{2}$-containing ceramics, Chen and Reyes Morel [6] reported that the constant $\psi$ in (1.3) may approach 2.0, which corresponds to $\mu=0.69$.

Based on the theory of deformation plasticity and normality flow rule, the multiaxial stress-strain relation for pressure-sensitive dilatant materials has been generalized by $\mathrm{Li}$ and Pan [8] from a power law hardening relation. The corresponding incremental constitutive relation can be found in Rudnicki and Rice [9] and [7].

From the viewpoint of phenomenological fracture mechanics, the initiation and growth of a crack depend on the surrounding stress and deformation fields near the tip. Therefore, analyses of the crack-tip stress and deformation fields are critical in relating continuum stress analyses to micromechanical failure mechanisms. The asymptotic crack-tip fields for the Mises materials have been presented by Hutchinson [1] and Rice and Rosengren [10]. The deformations of this well-known HRR fields are volume-preserving. An example of the HRR type crack-tip fields with volumetric deformation was presented by Hutchinson [11] for power-law creep materials undergoing creep-constrained grain boundary cavitation, and the dominance of this fields including the finite deformation effects was studied by Li, Needleman and Shih [12].

The hardening and non-hardening solutions of the crack tip fields for pressure-sensitive dilatant materials have been given by $\mathrm{Li}$ and $\mathrm{Pan}[8,13]$ under plane strain and plane stress conditions. The plane-strain perfectly-plastic near-tip field solution in [8] is an analytic solution. However, the plane-stress perfectly-plastic near-tip field in [13] was obtained by a numerical calculation using a Newton-Raphson scheme together with a combined fourth fifth-order Runge-Kutta integration scheme. In this study, a linear stress characteristic relation for the pressure sensitive material is derived. Hence, the analytic solution of plane-stress perfectlyplastic near-tip fields is obtained. Also, the relevant characteristic fields are presented. This perfectly plastic solution, containing $\mu$ as the pressure sensitivity parameter, is shown to correspond to the limit of the low-hardening solutions, and when $\mu=0$ it reduces to the perfectly-plastic near-tip field solution given by [1].

\section{The yield condition and the characteristic relation}

The yield condition for pressure-sensitive materials can be expressed as $\bar{\tau}_{e}+\mu \bar{\sigma}_{m}=\bar{\tau}_{0}$ or $\bar{\sigma}_{e}+\sqrt{3} \mu \bar{\sigma}_{m}=\bar{\sigma}_{0}$, where $\bar{\sigma}_{e}=\sqrt{3} \bar{\tau}_{e}$ is the effective stress, $\bar{\sigma}_{0}=\sqrt{3} \bar{\tau}_{0}$, and $\bar{\tau}_{0}$ is the shear yield stress. Referred to a Cartesian coordinates $\left(x_{1}, x_{2}\right)$, the yield condition for plane stress is written as

$$
f\left(\sigma_{i j}\right)=\sigma_{e}+\sqrt{3} \mu \sigma_{m}-1=\left(\sigma_{11}^{2}+\sigma_{22}^{2}-\sigma_{11} \sigma_{22}+3 \sigma_{12}^{2}\right)^{1 / 2}+\frac{\mu}{\sqrt{3}}\left(\sigma_{11}+\sigma_{22}\right)-1=0
$$

In (2.1) and throughout this paper, all unbarred stress quantities are normalized by $\bar{\sigma}_{0}$. 
A general development of the theory of perfect plasticity has been given by Hill [14, p. 132, p. 305]. This theory is used here to derive the stress characteristic relation for the pressure sensitive material under plane stress condition. Suppose that a curve $C$ is a stress characteristic and let $\sigma_{t}$ denote the normal stress component acting along the characteristic, $\sigma_{n}$ the normal stress component acting across the characteristic, and $\sigma_{t n}$ the shear stress. The condition for $C$ to be a stress characteristic is

$$
\frac{\partial f}{\partial \sigma_{t}}=\frac{2 \sigma_{t}-\sigma_{n}}{2 \sigma_{e}}+\frac{\mu}{\sqrt{3}}=0
$$

The yield condition requires that

$$
\sigma_{e}+\frac{\mu}{\sqrt{3}}\left(\sigma_{t}+\sigma_{n}\right)-1=0 .
$$

Combining (2.2) and (2.3) gives the following relation on a stress characteristic:

$$
\sigma_{t}=\frac{3+2 \mu^{2}}{2\left(3-\mu^{2}\right)} \sigma_{n}-\frac{\sqrt{3} \mu}{3-\mu^{2}}
$$

Let $\phi$ denote the angle, at some point $P$ on the characteristic $C$, between $C$ and the other characteristic, say $C^{\prime}$, rotated from $C$ to $C^{\prime}$ in the clockwise sense. With the help of the Mohr diagram, it is easy to show that

$$
\tan \phi=\frac{2 \sigma_{t n}}{\sigma_{n}-\sigma_{t}}
$$

Equation (2.5) indicates that the two characteristic directions coincide when $\sigma_{t n}=0$. Note that the bisectors of the two angles made by the characteristics at a point give the directions of the two in-plane principal stresses. It is evident from (2.2) that when the normality flow rule of plasticity is in force the tangents to a stress characteristic correspond to the directions of zero rate of extension and the stress characteristics coincide with the velocity characteristics.

Equation (2.4) is a general relation on the stress characteristics for materials that yield according to (2.1), and it will be used in the next section to derive the solution of the perfectly-plastic crack-tip stress fields.

\section{The perfectly plastic crack-tip stress fields}

We consider the planar crack depicted in Fig. 1, where the Cartesian coordinates $\left(x_{1}, x_{2}\right)$ and the associated polar coordinates $(r, \theta)$ are centered at the crack tip and the $x_{3}$ axis lies perpendicular to the $x_{1}-x_{2}$ plane.

Guided by the low hardening solutions for the pressure-sensitive materials in [13] and the perfectly plastic solution given by [1] for the Mises material for which $\mu=0$ in (2.1), we construct the stress fields under plane stress and mode I (or symmetric) loading condition with 


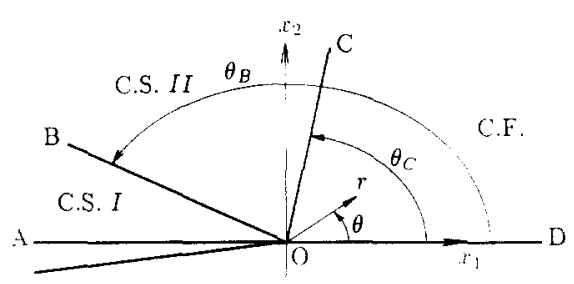

Fig. 1. The coordinates and the assembly of the crack-tip fields. The fields are composed of two constant stress (C. S.) sectors, and a centered fan (C. F.) sector.

the assumption that the material near the crack tip is yielding over the entire angular range. The crack-tip field is composed of a centered fan sector in front of the crack and two constant stress sectors with one of them adjacent to the traction-free crack face, see Fig. 1. At the boundary between the two constant stress sectors, i.e. at $\theta=\theta_{B}$, there is a jump in the radial stress $\sigma_{r r}$ coming from the quadratic yield condition. This discontinuity in $\sigma_{r r}$ is strongly suggested by our low hardening solutions [13], which will be briefly presented in Section 5. Some comments on stress discontinuity in perfect plasticity have been made in [1], and for more detailed discussion on this issue, see Hill [14, p. 157]. A general study of the structure of near tip fields for elastic-perfectly plastic materials can be found in Rice [15].

\subsection{The analytic solution in the centered fan sector $\left(0 \leqslant \theta \leqslant \theta_{C}\right)$}

For a perfectly plastic material, the stress near the tip is bounded. Hence, terms of the form $r \partial \sigma_{i j} / \partial r$ in the equilibrium equations must vanish as $r \rightarrow 0$. Therefore, the equilibrium equations reduce to two ordinary differential equations [15]:

$$
\begin{aligned}
& \frac{\mathrm{d} \sigma_{r \theta}}{\mathrm{d} \theta}=\sigma_{\theta \theta}-\sigma_{r r}, \\
& \frac{\mathrm{d} \sigma_{\theta \theta}}{\mathrm{d} \theta}=-2 \sigma_{r \theta} .
\end{aligned}
$$

In the fan sector, the radial lines emanating from the crack tip are characteristics, and, hence, $\partial f\left(\sigma_{i j}\right) / \partial \sigma_{r r}=0$. The characteristic relation (2.4) becomes

$$
\sigma_{r r}=\frac{3+2 \mu^{2}}{2\left(3-\mu^{2}\right)} \sigma_{\theta \theta}-\frac{\sqrt{3} \mu}{3-\mu^{2}}
$$

Equation (3.2) when $\mu=0$ reduces to $\sigma_{r r}=\frac{1}{2} \sigma_{\theta \theta}$ which is the characteristic relation for material of the Mises yield condition given by Hill [16].

Now, the yield condition can be replaced by the characteristic equation (3.2), which is combined with the two equilibrium equations in (3.1), reducing the nonlinear problem to the solving of linear ordinary differential equations for the three unknown stress components. The 
initial values of the stress components, $\sigma_{i j}(0)$, can be obtained from the yield condition, the characteristic relation (3.2), and the symmetry condition, i.e. $\sigma_{\mathrm{r} \theta}(0)=0$ for mode I loading. The results are

$$
\begin{aligned}
& \sigma_{r r}(0)=\frac{\left(3-\mu^{2}\right)^{1 / 2}-\sqrt{3} \mu}{\left(3-\mu^{2}\right)+\sqrt{3} \mu\left(3-\mu^{2}\right)^{1 / 2}} \\
& \sigma_{\theta \theta}(0)=\frac{2}{\left(3-\mu^{2}\right)^{1 / 2}+\sqrt{3} \mu}, \\
& \sigma_{r \theta}(0)=0 .
\end{aligned}
$$

Since there is no difficulty in solving of the linear O.D.E., in what follows we list the final results of the analytic solution in the centered fan sector.

$$
\begin{aligned}
& \sigma_{r r}=\frac{3+2 \mu^{2}}{\left(3-\mu^{2}\right)^{1 / 2}\left(3-4 \mu^{2}\right)} \cos k \theta-\frac{2 \sqrt{3} \mu}{3-4 \mu^{2}} \\
& \sigma_{\theta \theta}=\frac{2\left(3-\mu^{2}\right)^{1 / 2}}{3-4 \mu^{2}} \cos k \theta-\frac{2 \sqrt{3} \mu}{3-4 \mu^{2}} \quad\left(\mu<\frac{\sqrt{3}}{2}\right) \\
& \sigma_{r \theta}=\frac{1}{\left(3-4 \mu^{2}\right)^{1 / 2}} \sin k \theta, \\
& \sigma_{r r}=-\frac{2}{3} \theta^{2} \\
& \sigma_{\theta \theta}=\frac{2}{3}\left(1-\theta^{2}\right) \quad\left(\mu=\frac{\sqrt{3}}{2}\right) \\
& \sigma_{r \theta}=\frac{2}{3} \theta, \\
& \sigma_{r r}=-\frac{3+2 \mu^{2}}{\left(3-\mu^{2}\right)^{1 / 2}\left(4 \mu^{2}-3\right)} \cosh k \theta+\frac{2 \sqrt{3} \mu}{4 \mu^{2}-3} \\
& \sigma_{\theta \theta}=-\frac{2\left(3-\mu^{2}\right)^{1 / 2}}{4 \mu^{2}-3} \cosh k \theta+\frac{2 \sqrt{3} \mu}{4 \mu^{2}-3} \quad\left(\mu>\frac{\sqrt{3}}{2}\right) \\
& \sigma_{r \theta}=\frac{1}{\left(4 \mu^{2}-3\right)^{1 / 2}} \sinh k \theta .
\end{aligned}
$$

In (3.4) and (3.6),

$$
k=\left|\frac{3-4 \mu^{2}}{3-\mu^{2}}\right|^{1 / 2}
$$

where $|\cdots|$ denotes the absolute value. A direct substitution can verify that the solution (3.4) (3.6) satisfies the two equilibrium equations, the yield condition, and the symmetry condition, $\sigma_{r \theta}(0)=0$. 
Note that the solution in (3.4)-(3.6) is continuous with respect to $\mu$ on the entire interval $0 \leqslant \mu<\sqrt{3}$, especially at $\mu=\sqrt{3} / 2$. When $\mu=0$, (3.4) reduces to the solution for material of the Mises yield condition given by [16].

\subsection{The constant stress sector $I\left(\theta_{B}<\theta \leqslant \pi\right)$}

The traction-free conditions at $\theta=\pi$ and the yield condition (2.1) give the stress solution in this sector:

$$
\sigma_{11}=-\beta, \quad \sigma_{22}=\sigma_{12}=0
$$

or

$$
\sigma_{r r}=-\frac{1}{2} \beta(1+\cos 2 \theta), \quad \sigma_{\theta \theta}=-\frac{1}{2} \beta(1-\cos 2 \theta), \quad \sigma_{r \theta}=\frac{1}{2} \beta \sin 2 \theta,
$$

where

$$
\beta=\frac{\sqrt{3}}{\sqrt{3}-\mu} .
$$

3.3. The constant stress sector $I I\left(\theta_{c}<\theta<\theta_{B}\right)$

The continuity of traction across the boundary between the two constant stress sectors requires the continuity of $\sigma_{\theta \theta}$ and $\sigma_{r \theta}$ at $\theta=\theta_{B}$. The jump in $\sigma_{r r}$ across the radial boundary at $\theta=\theta_{B}$, resulting from the quadratic yield equation, is

$$
\begin{aligned}
\sigma_{r r}^{-}-\sigma_{r r}^{+} & =J\left(\theta_{B}\right) \beta \\
& =\frac{3}{3-\mu^{2}}\left[4-\left(3-4 \mu^{2}\right) \sigma_{\theta \theta}^{2}\left(\theta_{B}\right)-4\left(3-\mu^{2}\right) \sigma_{r \theta}^{2}\left(\theta_{B}\right)-4 \sqrt{3} \mu \sigma_{\theta \theta}\left(\theta_{B}\right)\right]^{1 / 2},
\end{aligned}
$$

where $J\left(\theta_{B}\right)$ can be calculated by substituting (3.9) into (3.11) to obtain

$$
J\left(\theta_{B}\right)=\frac{3}{2\left(3-\mu^{2}\right)}\left[1-\frac{4 \mu}{\sqrt{3}}+3 \cos 2 \theta_{B}\right]
$$

Taking into account the jump in $\sigma_{r r}$ as expressed in (3.11), gives the uniform stress in this sector

$$
\begin{aligned}
& \frac{\sigma_{r r}}{\beta}=\frac{1}{2}\left(J\left(\theta_{B}\right)-1\right)+\frac{1}{2}\left(J\left(\theta_{B}\right)-\cos 2 \theta_{B}\right) \cos 2\left(\theta-\theta_{B}\right)+\frac{1}{2} \sin 2 \theta_{B} \sin 2\left(\theta-\theta_{B}\right), \\
& \frac{\sigma_{\theta \theta}}{\beta}=\frac{1}{2}\left(J\left(\theta_{B}\right)-1\right)-\frac{1}{2}\left(J\left(\theta_{B}\right)-\cos 2 \theta_{B}\right) \cos 2\left(\theta-\theta_{B}\right)-\frac{1}{2} \sin 2 \theta_{B} \sin 2\left(\theta-\theta_{B}\right), \\
& \frac{\sigma_{r \theta}}{\beta}=-\frac{1}{2}\left(J\left(\theta_{B}\right)-\cos 2 \theta_{B}\right) \sin 2\left(\theta-\theta_{B}\right)+\frac{1}{2} \sin 2 \theta_{B} \cos 2\left(\theta-\theta_{B}\right) .
\end{aligned}
$$




\subsection{The determination of $\theta_{B}$ and $\theta_{C}$}

The continuity of traction across the radial boundary line between the centered fan sector and the constant stress sector II requires the continuity of $\sigma_{\theta \theta}$ and $\sigma_{r \theta}$ at $\theta=\theta_{C}$, which by the solutions (3.13) and (3.4)-(3.6) requires that

$$
\begin{aligned}
& \frac{1}{2}\left(J\left(\theta_{B}\right)-1\right)-\frac{1}{2}\left(J\left(\theta_{B}\right)-\cos 2 \theta_{B}\right) \cos 2\left(\theta_{C}-\theta_{B}\right)-\frac{1}{2} \sin 2 \theta_{B} \sin 2\left(\theta_{C}-\theta_{B}\right)=\sigma_{\theta \theta}^{C F}\left(\theta_{C}\right) / \beta \\
& -\frac{1}{2}\left(J\left(\theta_{B}\right)-\cos 2 \theta_{B}\right) \sin 2\left(\theta_{C}-\theta_{B}\right)+\frac{1}{2} \sin 2 \theta_{B} \cos 2\left(\theta_{C}-\theta_{B}\right)=\sigma_{r \theta}^{C F}\left(\theta_{C}\right) / \beta .
\end{aligned}
$$

In (3.14) and (3.15), $\sigma_{\theta \theta}^{C F}$ and $\sigma_{r \theta}^{C F}$ denote the solutions in the centered fan sector. A simple numerical scheme is used to solve (3.14) and (3.15) for the two unknowns, $\theta_{B}$ and $\theta_{C}$. The numerical values for $\theta_{B}$ and $\theta_{C}$ are listed in Table 1. For the Mises materials $(\mu=0)$, $\theta_{B}=151.24^{\circ}, \theta_{C}=79.84^{\circ}$. These numbers differ from those in [1] by less than $0.2^{\circ}$. Direct substitutions into (16) and (18) appearing in [1] can show that the two numbers given here are more accurate.

\subsection{The effects of pressure sensitivity on the near tip fields}

It is seen from Table 1 that when $\mu$ increases from 0 , the angle $\theta_{B}$ increases except for a small drop around $\mu=0.1$, while the angle $\theta_{C}$ always decreases. When $\mu$ closes to $\sqrt{3}, \theta_{B}$ approaches to $180^{\circ}$ and $\theta_{\mathrm{C}}$ approaches to $0^{\circ}$, indicating that the constant stress sector I adjacent to the crack face and the fan sector will become vanishingly small when $\mu \rightarrow \sqrt{3}$.

Figure 2 shows the perfectly-plastic stress fields for $\mu=0,0.6$ and 1.2 , calculated from the solution (3.4) (3.6), (3.9) and (3.13). It can be seen from this figure (also from (3.8) and (3.10)) that at the crack face $(\theta=\pi)$ a large $\mu$ gives a large uniaxial compressive stress. The major effects of the pressure sensitivity on the near tip fields is the change of the stress state directly ahead of the crack, at $\theta=0$. Since the stress state ahead of the crack is of major concern in studying crack initiation and growth, in Fig. 3 we plot the stress components $\sigma_{r r}$ and $\sigma_{\theta \theta}$ at $\theta=0$ versus the pressure sensitivity factor $\mu$. The points on these curves are calculated using (3.3). Figure 3 shows that the stress $\sigma_{r r}$ decreases with $\mu$, and becomes compressive when $\mu>\sqrt{3} / 2$ with a larger amplitude for a larger $\mu$, while the crack opening stress $\sigma_{\theta \theta}$ is always positive and decreases with $\mu$ until $\mu=3 / 2$. On the interval of $3 / 2<\mu<\sqrt{3}, \sigma_{\theta \theta}$ is elevated slightly with a limit value of $2 / 3$ when $\mu=\sqrt{3}$ is approached.

Table 1. The numerical values for $\theta_{B}$ and $\theta_{C}$ in degrees

\begin{tabular}{lrrrrrrrrrr}
\hline$\mu$ & 0.0 & 0.1 & 0.2 & 0.3 & 0.4 & 0.5 & 0.6 & 0.7 & 0.8 & 0.9 \\
$\theta_{B}$ & 151.24 & 151.01 & 151.14 & 151.54 & 152.16 & 152.96 & 153.92 & 155.02 & 156.25 & 157.61 \\
$\theta_{C}$ & 79.84 & 76.26 & 73.03 & 70.04 & 67.21 & 64.47 & 61.77 & 59.08 & 56.36 & 53.58 \\
\hline & & & & & & & & & & \\
\hline$\mu$ & 1.0 & 1.1 & 1.2 & 1.3 & 1.4 & 1.5 & 1.6 & 1.7 & 1.72 & 1.731 \\
$\theta_{B}$ & 159.09 & 160.71 & 162.49 & 164.44 & 166.63 & 169.15 & 172.21 & 176.66 & 178.15 & 179.57 \\
$\theta_{C}$ & 50.68 & 47.63 & 44.37 & 40.79 & 36.74 & 31.95 & 25.71 & 14.91 & 10.17 & 3.82 \\
\hline
\end{tabular}



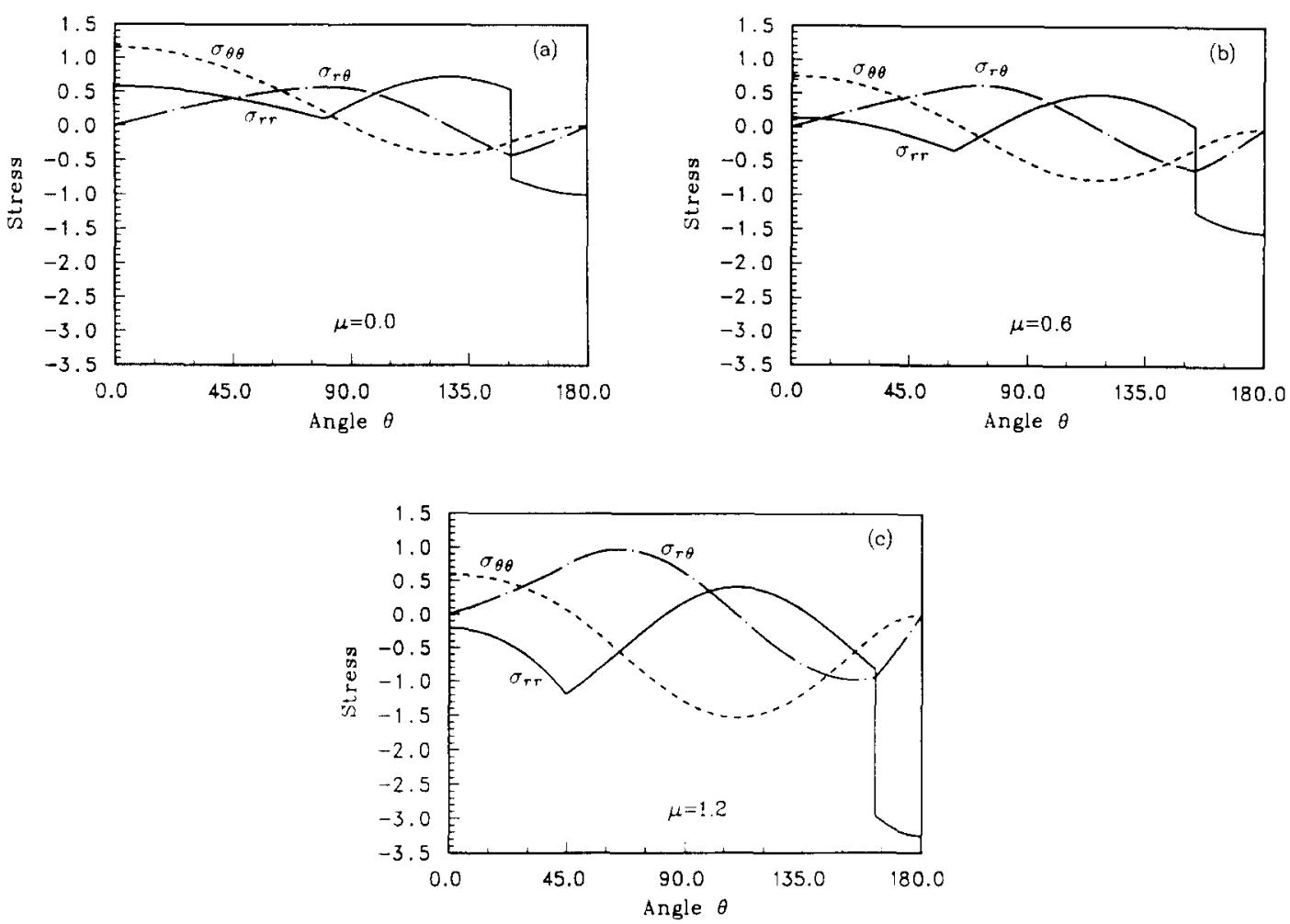

Fig. 2. The stress distributions for the perfectly plastic pressure-sensitive materials for $\mu=0,0.6$ and 1.2 .



Fig. 3. The effects of pressure sensitivity on the stress state ahead of the crack, at $\theta=0$. 


\section{The plane-stress characteristic fields}

In the centered fan sector, the radial lines emanating from the crack tip form the first family of characteristics. The second family of characteristics in the centered fan sector and the two families of characteristics in each of the two constant stress sectors can be determined from a known stress state by using (2.5) or through a coordinate rotation such that the stress characteristic relation (2.4) is met.

In the centered fan sector, $(2.5)$ becomes

$$
\tan \phi=\frac{2 \sigma_{r \theta}}{\sigma_{\theta \theta}-\sigma_{r r}},
$$

where $\phi$ is the angle rotated from the radial characteristics to the curved characteristics in the clockwise sense, so that the inclination of the curved characteristics is $(\theta-\phi)$. Substituting the solutions (3.4) $-(3.6)$ into (4.1) gives

$$
\begin{array}{ll}
\phi(\theta)=\arctan \left(\frac{2}{k} \tan k \theta\right), & \left(\mu<\frac{\sqrt{3}}{2}\right) \\
\phi(\theta)=\arctan (2 \theta), & \left(\mu=\frac{\sqrt{3}}{2}\right) \\
\phi(\theta)=\arctan \left(\frac{2}{k} \tanh k \theta\right) . & \left(\mu>\frac{\sqrt{3}}{2}\right)
\end{array}
$$

Integrating $\mathrm{d} r / r=-\mathrm{d} \theta / \tan \phi$ with $\phi$ expressed as in (4.2), gives the equations of the curved characteristics in the fan sector

$$
\begin{array}{r}
r^{2} \sin k \theta=\text { constant, } \quad\left(\mu<\frac{\sqrt{3}}{2}\right) \\
r^{2} \theta=\text { constant, } \quad\left(\mu=\frac{\sqrt{3}}{2}\right) \\
r^{2} \sinh k \theta=\text { constant. } \quad\left(\mu>\frac{\sqrt{3}}{2}\right)
\end{array}
$$

The characteristics in the two constant stress sectors are straight lines. In the constant stress sector I $\left(\theta_{B}<\theta \leqslant \pi\right)$, the inclination $\alpha$ of the straight characteristics satisfies

$$
\cos 2 \alpha=\frac{1}{3}\left(\frac{4 \mu}{\sqrt{3}}-1\right)
$$


The two solutions of (4.4), say $\alpha_{1}$ and $\alpha_{2}$, related by $\alpha_{1}+\alpha_{2}=\pi$, give the inclinations of the two families of characteristics in constant stress sector I. Some typical numbers are $\alpha_{1}=54.74^{\circ}$ for $\mu=0, \alpha_{1}=35.26^{\circ}$ for $\mu=\sqrt{3} / 2$, and $\alpha_{1} \rightarrow 0^{\circ}$ when $\mu \rightarrow \sqrt{3}$. In constant stress sector II $\left(\theta_{C}<\theta<\theta_{B}\right)$, one family of the characteristics are parallel to the radial line $O C$ with the inclination $\theta_{C}$, and the other family of characteristics has the inclination $\theta_{C}-\phi\left(\theta_{C}\right)$, where $\phi\left(\theta_{C}\right)$ is calculated according to (4.2).

Figure 4 shows the characteristic fields for $\mu=0,0.6$ and 1.2, where AOB and BOC are the two constant stress sectors, and $C O D$ is the centered fan sector. The angular locations of $O B$ and $\mathrm{OC}$, represented by $\theta_{B}$ and $\theta_{C}$, have been listed in Table 1 . Note that the maximum height of the characteristic patterns, i.e. the $y$-coordinate of point $\mathrm{B}$ in the three plots in Fig. 4 is the same. It can be seen from this figure that the pressure sensitivity factor $\mu$ generally tends to diminish the fan sector and the constant stress sector adjacent to the crack face.

\section{Concluding remarks}

In this study, a simple stress characteristic relation is obtained for pressure-sensitive perfectly plastic materials. This relation simplifies the nonlinear problem to the solving of linear ordinary differential equations. Hence, the analytic solution of the near-tip stress fields is derived. The mode I plane-stress crack-tip field solution when $\mu=0$ reduces to the stress field solution constructed by [1] for materials of the Mises yield condition.

When deformation theory of plasticity is invoked and the hardening behavior of the pressure-sensitive materials is described by a power law relation between the plastic shear strain $\gamma^{p}$ and the shear stress $\tau$ so that $\gamma^{p} \propto \tau^{n}$, the asymptotic stress and strain fields take the form

$$
\begin{aligned}
\sigma_{i j} & =\left[\frac{J}{C(n, \mu) r}\right]^{1 / n+1} \tilde{\sigma}_{i j}(\theta ; n, \mu), \\
\varepsilon_{i j} & =\left[\frac{J}{D(n, \mu) r}\right]^{n / n+1} \tilde{\varepsilon}_{i j}(\theta ; n, \mu),
\end{aligned}
$$
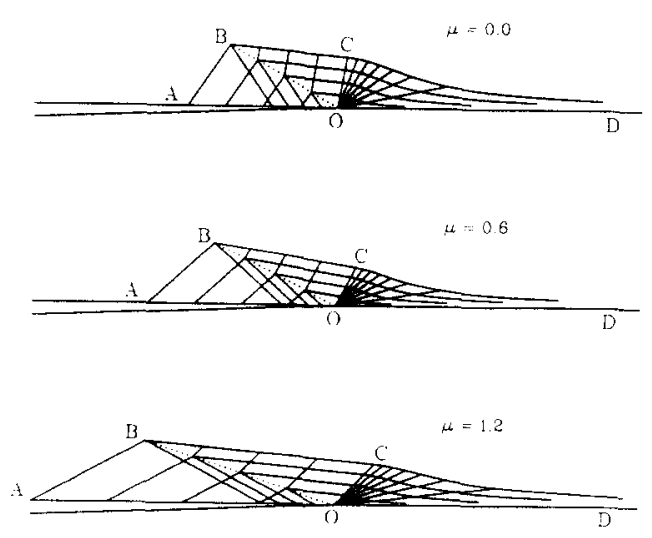

Fig. 4. The plane stress characteristic fields for $\mu=0,0.6$ and 1.2 . 
where $J$ is the path-independent $J$-integral introduced by Rice [17], and $r$ is the distance from the crack tip. The constant $C$ and $D$, and the angular functions $\tilde{\sigma}_{i j}$ and $\tilde{\varepsilon}_{i j}$ depend on the hardening exponent $n$ and the pressure sensitivity factor $\mu$. Details of the solution (5.1) for plane stress can be found in [13]. In Fig. 5, plotted are the angular distributions of $\tilde{\sigma}_{i j}$ for $n=20$ (low hardening material) and $\mu=0.0,0.6$ and 1.2. A comparison of Fig. 5 with Fig. 2 indicates that the perfectly plastic solutions obtained are indeed the limits of the corresponding hardening solutions as $n$ tends to infinity.

In [13], the hardening solutions for both stress and strain were given for the pressure-sensitive materials. We have shown that the perfectly plastic solution for stresses presented in this paper is the limit of the corresponding low-hardening solutions given in [13] as the hardening exponent $n$ tends to infinity. In this paper, no attempt has been made to obtain the plastic strain field for the pressure-sensitive materials, since the distribution of plastic strain near the tip according to perfect plasticity theory cannot be obtained by a simple analysis analogous to that for the stresses. However, certain details of the strain field can be inferred directly from the slip line field of Fig. 4. For example, the plastic strains can have a $1 / r$ singularity only in the centered fan region, $\theta<\theta_{c}$. According to the normality flow rule, the characteristic lines give the directions along which the extensional strain rates are zero. Therefore, in the fan zone, the radial component of strain must vanish for all $\theta$. These details of crack tip strain field are clearly reflected in the low-hardening strain solution $(n=20)$ in Fig. 6 of publication [13]. The perfectly plastic stress field obtained here for the pressure-sensitive materials when $\mu=0$ reduces exactly to the perfectly plastic stress field for the Mises materials given by [1]. The details and


Fig. 5. The angular distributions of the normalized stresses, $\tilde{\sigma}_{i j}$, obtained from the low hardening $(n=20)$ solutions for $\mu=0,0.6$ and 1.2 . 
comments made here on the plastic strain field for pressure-sensitive materials are similar to those on the plastic strain field for the Mises materials made by Hutchinson in his widely accepted paper [1].

It is found that when the pressure sensitivity factor $\mu$ is very large, the centered fan sector in front of the crack and the constant stress sector adjacent to the crack face will become very small. The major effects of the pressure sensitivity on the near-tip stress fields are the lowering of the normal stresses ahead of the crack. These effects can be used in studying the toughening mechanisms of polymeric and ceramic composite materials.

\section{Acknowledgement}

The support of this research by the College of Engineering of The University of Michigan is greatly appreciated.

\section{References}

1. J.W. Hutchinson, Journal of the Mechanics and Physics of Solids 16 (1968) 337-347.

2. D.C. Drucker, Metallurgical Transactions 4 (1973) 667-673.

3. W.A. Spitzig and O. Richmond, Polymer Engineering and Science 19 (1979) 1129-1139.

4. L.M. Carapellucci and A.F. Yee, Polymer Engineering and Science 26, No. 13 (1986) 920-930.

5. H.-J. Sue and A.F. Yee, Journal of Materials Science 24 (1989) 1447-1457.

6. I.-W. Chen and P.E. Reyes Morel, Journal of the American Ceramic Society 69, No. 3 (1986) 181-189.

7. A. Needleman and J.R. Rice, in Mechanics of Sheet Metal Forming, Donald P. Koistinen and Neng-Ming Wang (eds.), Plenum Publishing Corporation, New York (1978).

8. F.Z. Li and J. Pan, Journal of Applied Mechanics 57 (1990) 40-49.

9. J.W. Rudnicki and J.R. Rice, Journal of the Mechanics and Physics of Solids 23 (1975) 371-394.

10. J.R. Rice and G.F. Rosengren, Journal of the Mechanics and Physics of Solids 16 (1968) 1-12.

11. J.W. Hutchinson, Acta Metallurgica 31 (1983) 1079-1088.

12. F.Z. Li, A. Needleman and C.F. Shih, International Journal of Fracture 36 (1988) 163-186.

13. F.Z. Li and J. Pan, Engineering Fracture Mechanics 35, No. 6 (1990) 1105-1116.

14. R. Hill, The Mathematical Theory of Plasticity, Clarendon Press, Oxford (1950).

15. J.R. Rice, in Mechanics of Solids: The R. Hill 60th Anniversary Volume, H.G. Hopkins and M.J. Sewell (eds.), Pergamon Press, Oxford (1982) 539-562.

16. R. Hill, Journal of the Mechanics and Physics of Solids 1 (1952) 19-30.

17. J.R. Rice, Journal of Applied Mechanics 35 (1968) 379-386. 